\section{Cascade Reaction of 6-Deoxy-6-iodohexopyranosides Promoted by Samarium Diiodide: A New Ring Contraction of Carbohydrate Derivatives ${ }^{\dagger}$}

\author{
J ose Luis Chiara,* Sonia Martínez, and \\ Manuel Bernabé
}

Instituto de Química Orgánica General, C.S.I.C., J uan de la Cierva, 3, E-28006 Madrid, Spain

Received J une 13, 1996

Sequential transformations ${ }^{1}$ allow the assembly of complex molecules in a simple manner, with a minimum of purification steps, and are accordingly ideal components of elegant and efficient synthetic strategies. In recent years, samarium di iodide ${ }^{2}$ has evolved as a unique single electron reducing agent that is especially well suited to promote sequential processes that combine radical and anionic steps with a high degree of chemoand stereoselectivity. ${ }^{3}$ On the basis of the rich chemistry uncovered for this reagent, we hypothesized that its interaction with a 6-deoxy-6-iodohexopyranoside derivative A (Scheme 1) could trigger a reaction cascade that would eventually lead to a novel ring contraction ${ }^{4,5}$ of the pyranose moiety. This sequence requires four SET steps (i.e., 4 mol equiv of $\mathrm{Sml}_{2}$ ) and consists of (1) a reductive dealkoxyhalogenation to give the ring-opened hex-5-enal B; ${ }^{6}$ (2) an intramolecular ketyl-ol efin reductive coupling affording the ring-contracted organosamarium intermediate $\mathbf{C}^{3 a-c, 7}$ and (3) the intermolecular trapping of this organosamarium with appropriate electrophiles to produce finally the branched cyclopentitol derivative $\mathbf{D}$. $^{3 a-c}$

We report here the successful implementation of the postulated process for a series of 6-deoxy-6-iodohexopyranosides (1-7) substitution pattern and initial studies of the influence

${ }^{\dagger}$ A preliminary report of this work was presented at the XVIIth International Carbohydrate Symposium, Ottawa, J uly 1994; Abstract B1.67.

* To whom correspondence should be addressed. Fax: 34-1-5644853. E-mail: iqolc22@fresno.csic.es.

(1) For excellent accounts on the subject, see the recent Chem. Rev. thematic issue on "F rontiers in Organic Chemistry": Chem. Rev. 1996, 96 (1).

(2) For a recent review, see: Molander, G. A. Chem. Rev. 1996, 96, 307-338.

(3) (a) Molander, G. A.; Harris, C. R. J . Am. Chem. Soc. 1996, 118, 4059-4071. (b) Molander, G. A.; Harris, C. R. J . Am. Chem. Soc. 1995, 117, 3705-3716. (c) Molander, G. A.; McKie, J. A. J . Org. Chem. 1995, 60, 872-882 and references cited therein. (d) Enholm, E. J .; J iang, S.; Abboud, K. J . Org. Chem. 1993, 58, 4061-4069 and references cited therein. (e) Curran, D. P.; Wolin, R. L. Wolin, R. L. Synlett 1991, 317318. (f) Curran, D. P.; Fevig, T. L.; Totleben, M. J . Synlett 1990, 773774.

(4) For a recent highlight on ring contractions in carbohydrates, see: Redlich, H. Angew. Chem., Int. Ed. Engl. 1994, 33, 1345-1347.

(5) For a related ring contraction of carbohydrate derivatives using

$\mathrm{Sml}_{2}$, see: Chénedé, A.; Pothier, P.; Sollogoub, M.; Fairbanks, A. J .;

Sinay, P. J . Chem. Soc., Chem. Commun. 1995, 1373-1374.

(6) Originally described with $\mathrm{Zn}$ or butyllithium by: (a) Bernet, B.; Vasella, A. Helv. Chim. Acta 1979, 62, 1990-2016. For modified procedures, see: (b) Fürstner, A.; Baumgartner, J .; J umbam, D. N. J . Chem. Soc., Perkin Trans. 1 1993, 131-138 and references cited therein. $\mathrm{F}$ or previous reports on ring scissions of cyclic $\beta$-halo ethers promoted by samarium diiodide, see: (c) Crombie, L.; Rainbow, L. J . J. Chem. Soc., Perkin Trans. 1 1994, 673-687. (d) Tetrahedron Lett. 1988, 29, 6517-6520.

(7) (a) Fevig, T. L.; Elliot, R. L.; Curran, D. P. J . Am. Chem. Soc. 1988, 110, 5064-5067. (b) Enholm, E. J .; Trivellas, A. Tetrahedron Lett. 1989, 30, 1063-1066. (c) Baldwin, J. E.; Turner, S. C. M.; Moloney, M. G. Tetrahedron 1994, 50, 9411-9424.

(8) All new compounds showed correct microanalytical and spectroscopic data.
Scheme $1^{\text {a }}$

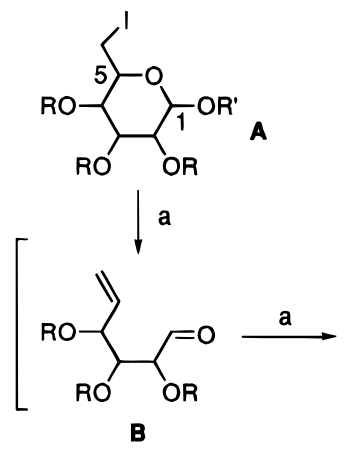

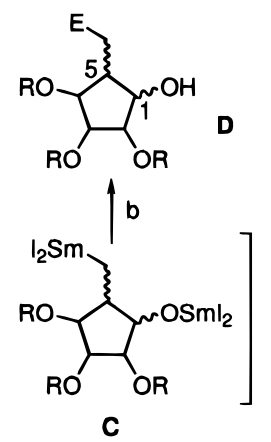

a Reagents: (a) $2 \mathrm{Sml}_{2}$; (b) (i) $\mathrm{E}^{+}$; (ii) $\mathrm{H}^{+}$.

Table 1. Reaction of 6-Deoxy-6-iodohexopyranosides with $\mathrm{Sml}_{2}$ in THF-HMPA

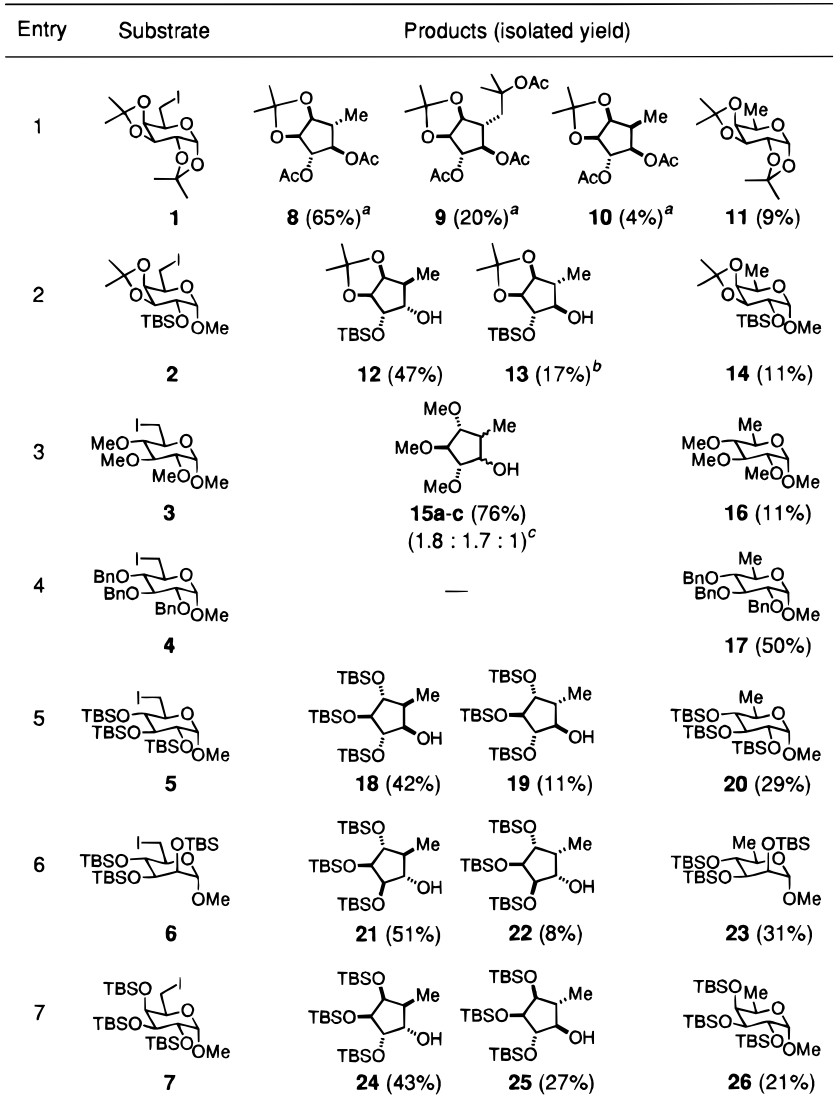

a Products obtained after acetylation of the crude reaction mixture. ${ }^{b}$ The stereochemistry of $\mathbf{1 3}$ was further confirmed by its transformation into 8 by desilylation (TBAF in THF) followed by in situ acetylation ( $\mathrm{Ac}_{2} \mathrm{O}$, pyridine). ${ }^{\mathrm{C}}$ Inseparable mixture. Ratio determined by ${ }^{1} \mathrm{H}$ NMR.

of the reaction conditions and protecting groups on the outcome of the reaction.

Preliminary experiments were performed with compound $\mathbf{1}$ to determine the optimum reaction conditions. In a typical procedure, a $0.05 \mathrm{M}$ solution of $\mathbf{1}$ in THF was

(9) Compounds 1-4 were prepared in high yield by iodination of the corresponding partially O-protected pyranosides with the triphenyl phospine/imidazole/iodine reagent. ${ }^{10}$ Compounds 5-7 were prepared in high yield by silylation of the corresponding methyl 6-deoxy6-iodopyranosides ${ }^{11}$ with TBDMSOTf in 2,6-lutidine/ $\mathrm{CH}_{2} \mathrm{Cl}_{2}$.

(10) Garegg, P. J .; Samuelsson, B. J . Chem. Soc., Perkin Trans. 1 1980, 2866-2869.

(11) Whistler, R. L.; Anisuzzaman, A. K. M. Methods Carbohydr. Chem. 1980, 8, 227-231.

(c) 1996 American Chemical Society 
added dropwise to a $0.1 \mathrm{M}$ solution of $\mathrm{Sml}_{2}$ ( $6 \mathrm{~mol}$ equiv) in THF and HMPA (30 mol equiv) at $22{ }^{\circ} \mathrm{C}$, and the mixture was stirred for $1.5 \mathrm{~h}$. In the case of $\mathbf{1}$, the reaction products were better isolated after in situ acetylation of the crude reaction mixture $\left(\mathrm{Ac}_{2} \mathrm{O} /\right.$ pyridine). Three cyclopentane products $(\mathbf{8}-\mathbf{1 0})^{8,12}$ were isolated together with the cor responding 6-deoxypyranoside (11). Cyclopentane 9, which was shown to have the same stereochemistry at the two new stereocenters as the major cyclopentane $\mathbf{8}^{12}$ probably derives from attack of the major diastereoisomeric organosamarium intermediate $\mathbf{C}$ on the acetone molecule released from $\mathbf{1}$ in the reductive elimination step. Lower temperatures favored formation of $\mathbf{1 1}\left(12 \%\right.$ at $0{ }^{\circ} \mathrm{C} ; 15 \%$ at $-25^{\circ} \mathrm{C} ; 63 \%$ at -78 $\left.{ }^{\circ} \mathrm{C}\right)^{13}$ and produced a decrease in the yield of $\mathbf{9}$ (3\% at 0 ${ }^{\circ} \mathrm{C}$; $0 \%$ at $-25{ }^{\circ} \mathrm{C}$ or below) without having a significant influence on the diastereoselectivity of the ring-closure reaction. Also important is the influence of the nature of the protecting groups both on the diastereoselectivity (cf. Table 1, entries 1 and 2 and 3 and 5) and on the extent of the competing simple dehalogenation reaction. The highest diastereoselectivity was observed for the transformation of substrate $\mathbf{1}$ and is probably the result of electrostatic repulsion in the intermediate ketyl radical anion derived from B, where $0-2$ is presumably in the form of a samarium(III) alkoxide. Increasing steric bulk around the halogenated carbon produced an increase in the amount of the simple dehalogenation reaction giving the 6-deoxypyranoside (cf. Table 1, entries 1 and 7 and 3 and 5). ${ }^{14}$ Interestingly, in the case of the benzylated derivative 4 the only isolable product was the corresponding 6-deoxypyranoside 17 (Table 1, entry 4). ${ }^{15} \mathrm{~A}$ higher yield of $\mathbf{1 7}(70 \%)$ was obtained when this reaction was performed in the presence of $\mathrm{MeOH}$ (30 equiv). In most cases, the cyclic products have the methyl group trans to the hydroxyl group, as expected for these exo cyclizations. $^{3}$ In addition, a trans orientation between the hydroxyl and the vicinal alkoxy group at C-2 has been observed in all cases, except in the cyclizations of the silylated galacto derivatives $\mathbf{2}$ and $\mathbf{7} .{ }^{16}$

Further efforts were directed at determining some mechanistic aspects of this reaction cascade. Thus, compound $\mathbf{1}$ was subjected to the standard reaction conditions but 10 equiv of $\mathrm{D}_{2} \mathrm{O}$ was added to the $\mathrm{Sml} \mathrm{L}_{2}$ solution just prior to the addition of $\mathbf{1}$. To our surprise, under these conditions two new cyclopentanes (27 and 28) ${ }^{17}$ were obtained together with 8 (which was no longer

(12) The stereochemistry of the two new centers was determined by ${ }^{1} \mathrm{H}$ NMR and 2D NOESY studies (see the supporting information).

(13) A similar effect of temperature on the extent of competing dehalogenation vs dealkoxyhalogenation has been previously observed in the reaction of halo sugars with metal-graphite reagents: F تurstner, A.; Kogl bauer, U.; Weidmann, H. J . Carbohydr. Chem. 1990, 9, 561570. Fürstner, A.; J umbam, D.; Teslic, J .; Weidmann, H. J . Org. Chem. 1991, 56, 2213-2217.

(14) This sensitivity of dealkoxyhalogenation reactions to steric effects has also been observed in the reaction of metal-graphite reagents with furanose systems. ${ }^{6 b}$

(15) Probably in this case a 1,5-hydrogen migration from the benzyl group at 0-4 to C-6 is competing favorably with the reduction of the intermediate primary radical at C-6 by $\mathrm{Sml}_{2}$. Similar 1,5-hydrogen migrations in radical intermediates from benzylated sugar derivatives have been reported before: (a) Liotta, L. J .; Bernotas, R. C.; Wilson, D. B.; Ganem B. J . Am. Chem. Soc. 1989, 111, 783-785. (b) Martin, O. R.; Xie, F.; Kakarla, R.; Benhamza, R. Synlett 1993, 165-167. (c) Barbaud, C.; Bols, M.; Lundt, I.; Sierks, M. R. Tetrahedron 1995, 51, 9063-9078 (see also ref 6b).

(16) A similar trans-directing effect of adjacent alkoxy groups has been observed in intramolecular pinacol coupling reactions promoted by samarium diiodide, and it was attributed to steric and/or electrostatic interactions involving the ketyl radical anion and the alkoxy substituent: Chiara, J . L.; Cabri, W.; Hanessian, S. Tetrahedron Lett. 1991, 32, 1125-1128.

\section{Scheme 2}

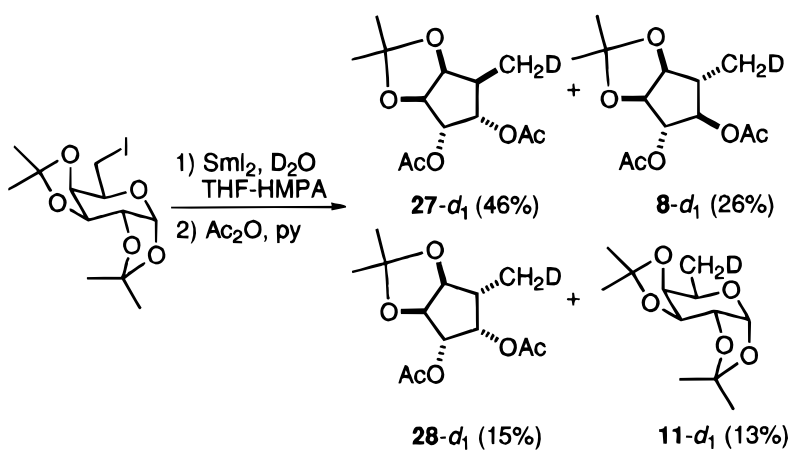

the major product) and the dehalogenated product $\mathbf{1 1}$ (Scheme 2), all showing deuterium incorporation at the methyl group as determined by ${ }^{1} \mathrm{H}$ and ${ }^{13} \mathrm{C}$ NMR. ${ }^{18}$ The change in diastereoselectivity induced by the proton source is unprecedented and could be the result of protonation of the 0-2 samarium(III) alkoxide in the ketyl radical anion intermediate derived from $\mathbf{B}$. Che lation of samarium(III) or intramolecular hydrogen bonding in this radical anion intermediate may account for the predominant formation of 27.3c,19,20 The presence of deuterium in the final products indicates the intermediacy of alkyl anions both in the reductive elimination reaction and in the carbocyclization reaction. $6 c, 3 b$ In contrast, when the proton source was added immediately after complete consumption of the starting material no deuterium incorporation took place. ${ }^{3 a}$

In conclusion, the process described in this paper represents a new and simple method for the one-pot preparation of highly functionalized, enantiomerically pure cyclopentanes from readily accessible carbohydrate derivatives. ${ }^{21}$ F urther efforts directed to the trapping of the final organosamarium intermediate with different electrofiles are in progress.

Acknowledgment. Financial support by DGICYT (Grant No. PB93-0127-C02-01) is gratefully acknowledged.

Supporting Information Available: General experimental procedure for the reaction of 6-deoxy-6-iodohexopyranosides with $\mathrm{Sml}_{2},{ }^{1} \mathrm{H}$ and ${ }^{13} \mathrm{C}$ NMR data, and 2D NOESY cross-peak intensities for cyclopentane products (4 pages).

\section{J 0961116+}

(17) Compound $\mathbf{2 7}$ has the same stereochemistry as 12. Thus, the latter could be transformed into $\mathbf{2 7}$ by desilylation (TBAF in THF) followed by in situ acetylation ( $\mathrm{Ac}_{2} \mathrm{O}$, pyridine).

(18) Deuterium incorporation was almost complete (>90\%) for the cyclopentane products but only $70 \%$ for the dehal ogenated product 11 as determined by ${ }^{1} \mathrm{H}$ NMR. This result suggests that formation of $\mathbf{1 1}$ is taking place via both one-electron (by hydrogen atom abstraction, probably from solvent) and two-electron (by protonation of an organosamarium intermediate) processes. See, however, ref $3 a$.

(19) This change in diastereoselectivity could also be due to formation of a different samarium complex in the presence of water. Water has been shown to enhance the reducing ability of $\mathrm{Sml}_{2}$. Hasegawa, E.; Curran, D. P. J . Org. Chem. 1993, 58, 5008-5010. Likewise, it has been reported recently that simple al cohols also bind strongly to Sml and that this binding affects the chemoselectivity of reductions of olefins by this reagent. Yacovan, A.; Hoz, S.; Bilkis, I. J . Am. Chem. Soc. 1996, 118, 261-262.

(20) Protonation of the oxygen of the ketyl radical anion by water is unlikely: $\alpha$-hydroxyalkyl radicals are strong acids with $\mathrm{pK}_{\mathrm{a}}=10.7-$ 12.1. Neta, P. Adv. Phys. Org. Chem. 1976, 12, 223-297. Molander has not observed any effect of proton sources on the diastereoselectivity of ketyl-olefin radical cyclizations in simple systems. Molander, G. A.; McKie J . Org. Chem. 1992, 57, 3132-3139.

(21) When this paper was in preparation, a report appeared in the literature describing the cyclization of carbohydrate-derived hex-5enals promoted by Sml 2: Cronjé, J .J .; Holzapfel, C. W.; Williams, D. B. G. Tetrahedron Lett. 1996, 37, 1305. 